\title{
The Synergetic Approach to Liberal Education of the University Students
}

\author{
Larisa I. Tararina \\ Elena E. Sokolova \\ Elizaveta V. Limarova
}

Russian State Social University, Moscow, Russia

E-mail: It31@mail.ru

Larisa N. Ivanova

Chuvash State Pedagogical University named after I.Y. Yakovlev, Cheboksary, Russia

Svetlana N. Fedorova

Alexey G. Oshaev

Mari State University, Yoshkar-Ola, Russia

Sergey A. Chernov

Chuvash State Pedagogical University named after I.Y. Yakovlev, Cheboksary, Russia

Elmira R. Khairullina

Kazan National Research Technological University, Kazan, Russia

Doi:10.5901/mjss.2015.v6n2s3p98

\section{Abstract}

The thematic justification of this problem is driven by the emergence of a new stage in the development of liberal education of the students, where the most important idea is the synergy - the interdisciplinary field of research that sets its strategic objective to employ the limelight principles underlying the processes of students self-organization while studying the liberal arts. The purpose of the article is theoretical and methodological underpinning of the synergetic approach to liberal education of the students. The paper presents the theoretical foundations of the synergetic approach to liberal education in university that are expressed in the key concepts; in a trinity of the educational purposes-attractors; in the principles keeping the continuity of traditional and synergetic ideas; the functions determined by the essence and characteristic synergistic indications of liberal education; in the structure and content of the synergetic model of liberal education; in pedagogical conditions of employing the synergistic approach in liberal education (basic components of liberal education of the students). The article submissions may be useful for the specialists in the field of synergy, the researchers in the system of continuous professional education, the academic staff of the universities. The research is also recommended for the undergraduates, graduate students and students of universities specialized in research, the students of the continuous education and retraining.

Keywords: synergetic approach; self-organization; synergetic principles; synergetic technologies; interdisciplinary teaching module.

\section{Introduction}

\subsection{Background}

The development, use and further growth of high technologies in the areas of domestic production, the introduction of computer and information technology have set a compulsory objective of educating a new type of specialists, intellectual professionals who are competent in relevant professionally-important competencies, personal culture and education in the liberal arts. To achieve the objectives of the Federal State Standards of higher vocational education (FSES ) in liberal 
education the problem of implementing the innovative concepts, approaches and paradigms emerges actual. The specialists (Andreev, 1996; Budanov, 2013; Vyugina, 2015; Zeer, 2000; Novikov, 2000) have found that the most promising, notable approach to liberal education of the future specialists of high-tech industry is now becoming a synergistic approach as a methodological principle of learning the mechanisms of the students' and teachers' selfdevelopment, self-organization of the scientific knowledge focused on multi-dimensional, multi-component and polyphonic (alternative and variable) knowable processes of modernization of liberal education, on discovering the unsolved or unrevealed conditions that are intended for openness, co-creation, self-development, awareness of the contingent probability in their development. These directions prevail since they create an educational environment for liberal education of the students that anticipates the requirements of FSES of higher vocational education and the labor market demand in creative, self-actualized specialists. The pragmatic interpretation of the synergetic approach to liberal education involves the enriching the teaching process with the ideas of self-organization and self-development of the students in the process of designing and implementing the interdisciplinary teaching modules of the humanities.

\subsection{The theoretic and methodological novelty of the problem}

The existing educational concepts of liberal education in universities and their identical organizational structures of liberal education lose their effectiveness and ability to act as the leading areas of teaching theory and practice. These trends are largely conditioned by the lack of scientifically-grounded approaches to the development of innovative, theoretical and methodological foundations of liberal education, reconsidering the content and meaning of the key concepts, the essence, the principles, the functions and conditions for eliciting their educational potential, capable to provide selforganization and self-development of the student's individuality while studying the humanities. The requirements of the FSES in vocational education operating in the educational practice of higher school state the necessity of rapid development of the conceptual approaches to modernization of liberal education in which the identity of the student and his full, harmonious, universal holistic, creative development becomes a systemically-important factor. According to the results achieved by the leading specialist in the pedagogy theory and practice (Andreev, 1998; Makhmutov, 1993; Novikov, 2000; Talanchuk, 1993) the design and implementation of the theoretical and methodological foundations of the conceptual approaches involve: reconsidering the discursive content of the conceptual and categorical apparatus of liberal education enriched with synergy ideas; the definition and identification of the objectives, principles, functions and pedagogical conditions for self-transformation and self-development of the students in liberal education, design and development of the interdisciplinary teaching modules of the humanities and other types of curriculum papers.

\subsection{Pragmatic relevance}

The practical relevance involves updating and rethinking of the structure and content of liberal education through implementing a synergetic model in education, using the courseware of the liberal arts based on the interdisciplinary teaching modules, programs, methodical projects of the scientific support of liberal education.

\subsection{Literature Review}

The theoretical and methodological foundations of the synergetic approach to students liberal education lie on the research of the specialists, structured according to their application: a philosophical approach (Zapesotsky, 2003; Prigozhin, 1986; Haken, 1984); the methodological foundations of synergy in education (Andreev, 1996; Vinenko, 2001; Knyazeva, 1991); the concept of self-organization in pedagogy (Boguslavskiy, 1999; Budanov, 20013; Fedorova, 1997).

The theoretical foundations of the synergetic approach to liberal education of the students are expressed in the contents of the key concepts (synergetic approach, self-organization, synergetic principles, synergistic technologies, interdisciplinary teaching module), the trinity of the liberal arts educational purposes (the strategic one is forming liberal education awareness and individual culture of the student, the tactical one is developing readiness of an individual to selforganization and self-transformation, the operational one means formation of the competences of the individual selforganization in the process of studying the humanities), in principles that preserve the continuity of the connections between traditional and synergetic ideas (conceptuality, systemacity, fundamentality, integrity, innovation, hierarchy, homeo-statics, nonlinearity, instability, incompleteness, emergence, self-actualization), in the functions determined by the synergistic essence and its performance in liberal education (methodological, self-organizational and synergetic contact interaction between the teacher and the student, professional and personal focus of liberal education on individual student's adaptability to the learning process, information support of the liberal arts education), in structure and content of 
a synergetic model of the students' liberal education, in pedagogical conditions of the synergetic approach realization (basic components of liberal education, synergistic technologies, synergetic project of the curriculum papers, criteria for the evaluation of the synergetic approach productivity in liberal education).

\subsection{The methods of research}

To achieve the goals and objectives of the study we have involved a range of methods: the analysis of philosophical, pedagogical, psychological and methodological literature considering the classification within a reasonable set of parameters; studying and generalization of mass and advanced pedagogical experience in liberal education of the university students; observation of the students' educational activities and obtaining quantitative indicators, written and oral surveys; the analysis of traditional and innovative syllabi, teaching modules, the coursebooks and teaching aids. The complex of the diagnostic procedures include: pedagogical observation of the educational process; testing the students and the liberal arts teachers; a natural pedagogical assessment (summative and formative); designing and testing of the interdisciplinary modules based on synergetic principles of self-organization and self-transformation and encompassing a complex of content-related, technologic and criteria-based devices; problem solving; business games; statistical and analytical data processing of the experiment; the Delphi technique.

\subsection{The performance and criteria of the synergetic approach efficiency in students' liberal education}

The indicators: availability, depth, the range of liberal, scientific, general professional, special and practical knowledge joint with the students' experience.

The criteria:

- the knowledge (advanced knowledge and proficiency in a particular specialty, philosophical, political, historical, literary-linguistic, psycho-pedagogical, legal, artistic and creative and other basic knowledge, its system, a high level of generalization that meets the requirements of education, high personal culture, social and professional competence);

- the needs (in communication, self-realization, understanding, cognition, self-respect, self-determination, reflection, understanding the meaning of life);

- the ability to generate new ideas and use non-standard work methods, to transfer knowledge and modes of behavior to new non-standard situations in professional, socio-cultural, family and home life;

- the axiological orientations (worldview, vocational, inventive, creative), norms, ideals, values, judgments couched in clear terms, students' opinions, behaviors in the course of work on research projects.

\section{Methodological Framework}

\subsection{The synergetic discourse of the key concepts.}

The results of the research have allowed us to specify and rethink the key concepts of the content of the students' liberal education in university within theoretical and methodological positions of the synergetic approach:

- The synergetic approach is a theoretical and methodological strategy focused on the implementation of the advanced objectives, principles, functions, content, technologies of the liberal education of the student's personality as a self-organizing and developing system capable of reflection and self-transformation which allows to rethink the particularities of the students' liberal education, to create new conditions to elicit their creative abilities, to thoroughly evaluate the changing variety of the principles, ways and methods of developing a creative activity in the course of liberal education, to set the process of students liberal education free from unilinearity and dogmas which allow for multifunctionality and multidimensionality of the educational theories and hypotheses;

- Self-organization is a self-consistent functioning of the student as a subject of study through direct communication and feedback from the external environment. The environment at this stage of research is represented by the process of liberal education of the students that is capable of self-transformation and selfdevelopment due to such characteristics as dynamics, interdisciplinarity, informativity;

- The synergetic principles are the principle of acknowledgement of an inherent value of each individual; the principle of fluctuations (deviations) of creative thinking; the principle of contradictory process of liberal education; the principle of dissipation (self-forming) of the creative abilities as a core of liberal education; the 
principle of a single tempo-world (the development rate of the student and the teacher); the principle of the age-dependent sensitivity (opening and closing opportunities of the individual student where the moment of opening is the moment of truth, of sensitivity);

- The synergistic technologies - the synergy of the technologies is primarily determined by the fact that they provide transition from the arrangement of educational process by a teacher to the transformation and organization of the process by the trainees themselves. These processes are based on the changes of the axiological attitude of the students to acquiring liberal education as to the process of self-discovery, selfdetermination while reconsidering the presented educational information. All the educational activities of the student appear as a creative activity which is characterized by three types of operations: logical, intuitive, heuristic. In the technologic process of self-organization and self-education the educators solve the problems of liberal education mediated by the choice of identical technologies: at the level of the curriculum these are the technologies of the educational process arrangement (lectures and seminars, interactive, problem-based, design-based, modular competence-based ones); at the level of cyclic goals suppressing any variation in the curriculum and programs which have arisen under fluctuations (the external influences on the process of the liberal education development), effective technologies of the theoretical, practical and industrial training: modular, design, online, context, and of other fields. The specific course objectives are implemented in the intermediate training technologies taking into account the characteristics of the studied subject. The objectives of individual activities involve the use of certain technologies: the formation of the competencies of selftransformation, self-organizing, self-learning, critical thinking, analysis and introspection of the behavior and activity, decision-making, arguing own position, etc.;

- the interdisciplinary teaching module - a constructive interdisciplinary project, an organized form of interaction of the humanities, scientific, professionally relevant disciplines to understand, study and manage the hypercomplex systems phenomena: environmental problems, globalization, crisis management, social engineering, artificial intelligence problem, the integration of psychology and medicine, space exploration and others. In liberal disciplines the study of the interstate conflicts is an interdisciplinary project of proving the hypothesis version and its causes. The interdisciplinary teaching module in liberal education is designed to teach within organizational and methodological structure of academic disciplines as a bridge between the separated subjects, as a route in a complex landscape of disciplinary discourses if its purpose is research and descriptive activities. But the main purpose of the interdisciplinarity is communication or communication selforganization. Hence there is the introduction of interdisciplinary methodology, transdisciplinary norms, values, invariants and universals into the scientific worldview (Budanov, 2013).

\subsection{The semantic content of interdisciplinarity}

The researchers in the field of synergy (Budanov, 2013) have described five types of interdisciplinary strategies and the definition of "interdisciplinarity" in accordance with these five types:

- the interdisciplinarity as harmonization of concepts related to the related disciplines. It is expected to construct the common phenomenological framework for the related disciplines, where every discipline uses its thesaurus. An example is the interdisciplinary courses of physical chemistry, biochemistry, sociology and psychology;

- the interdisciplinarity as transcoordination of the concepts of the disciplines that are not closely related basing on the relations of the general scientific, invariant, universal methods of system analysis and synergy. This type of interdisciplinarity is used within a variety of disciplines;

- the interdisciplinarity as a heuristic hypothesis is an analogy that shifts the construction of one discipline to another without sufficient justification at first. The incompleteness and creativity of such hypothetical shifts calls for either reasoning within this discipline or revising the grounds for the shift. For example, the pilotwaves in quantum theory being introduced to explain the phenomena of wave-corpuscle duality have not been adapted to it, but the random waves are generally recognized in modern science. They have unfixed all established notions of a modern human about quantum ontology;

- the interdisciplinarity as a constructive interdisciplinary project, organized form of the interaction of many disciplines to understand, study and manage the phenomena of hypercomplex systems. Today it's environmental problems, global studies, crisis management, social engineering, artificial intelligence problem, the integration of psychology and medicine, space exploration, etc. ;

- the interdisciplinarity as a communication network or communication self-organization. On the basis of 
communication network they introduce the interdisciplinary methodology, transdisciplinary norms and values, the invariants and universals of the scientific worldview, develop synergy and system analysis, fashion and gossip in society.

The research has shown that the identified definitions of interdisciplinarity according to their semantic content correspond to the modern trends in higher educational process transformations and confirm their importance for the implementation of the synergetic approach in liberal education.

2.3 The pedagogical conditions of designing and implementing the interdisciplinary teaching modules in students' liberal education

- At the level of content - an organized form of interaction between the humanities, harmonization of concepts, self-organization of communication, heuristic analogy hypothesis that transfers the design of one discipline to another basing on the principle of self-organization;

- At the procedural level - the modification of interaction between the teacher and students, expanding partnerships, cooperation and mutual assistance;

- At the level of teaching aids - the interactive and design technologies;

- $\quad$ At the level of educational result - the students readiness to self-organization and creative activity;

- At the level of conditions - the collective creative environment in liberal education generating and supporting creative individual behavior, initiating its self-actualization;

- At the level of liberal education proficiency - availability and depth of knowledge, knowledge competence; maintenance and development of the cultural requirements; the system of axiological orientations and social norms being the norms of various fields of activity; ideals; choice of liberal alternatives; cultural and ethnocultural identity; degree of involvement into learning and cognitive, social and professional activities.

\section{Results}

\subsection{The interdisciplinary teaching module as a didactic construct.}

This construct is founded on the guiding principles of design and problem-based developmental education enriched with the ideas of synergistic approach, self-organization and self-transformation in liberal education. Every module provides a detailed description of the activity-related, didactic purposes confirmed by the materials content being studied and the specific diagnostic and design tasks. The content of the educational material is a complete unit of educational material supplemented with methodological instructions, innovative knowledge control system allowing you to quickly adjust the teaching process. The goal - attractor of the educational process is focused on the formation of students' readiness for self-transformation, self-organization and self-development.

3.2 The structure of the requirements for the design and implementation of the interdisciplinary teaching module in students' liberal education:

- The presence of a problem significant for the research and creative activities, requiring the integrated knowledge, exploratory search for the solution (for example, study of the problem of personal immunity in different regions of the world, the creation of a series of reports from around the globe on the issue of intercultural communication);

- The hypothetical, theoretical, cognitive relevance of the expected results (for example, a report to the appropriate services about the level of the society general culture, about the factors affecting the state of culture, the trends in the development of this problem, a joint newspaper issuing according to specific problems, an Almanac with the play-by-play reports; an international draft law on protection of the cultural monuments, historic events in different regions; a video showing the problem and the ways of its solution etc );

- The independent (individual, pair and group) activity of the students;

- Phasing of the content part of the interdisciplinary teaching modules (with indication of the intermediate results);

- The use of research methods that involve a sequence of actions:

- The determination of the problem and the research tasks arising from it while implementing the joint method of "brainstorming" and design technologies; 
- Hypothesizing and its solution;

- The discussion of the research methods (the analysis of information, facts, statistical and experimental methods, observation etc. );

- The discussion of the ways to formalize results (presentation, role play, video, report and others. );

- The collection, systematization and analysis of the data obtained;

- Summarizing, formalizing of the results and their presentation;

- $\quad$ The conclusions, bringing up new research problems;

\subsection{The Methodological materials to help the students}

- At the heart of each module there is a problem significant for the participants. No problem - no activity.

- Before starting to work on the module project try to answer the following questions:

- Why is participation in this activity important for me? Why are we doing this project?

- What do I need to do to implement the plan? How exactly can we do it? What should we start with? What should happen in the end?

- The stages of designing an interdisciplinary module are held according to the scenarios of design techniques:

1. Immersion in the problem. Problem formulation. Goal-setting and tasks.

2. The organization of the activities. Organization of the group work, distribution of the roles for every participant in the group. Planning of joint and individual activities to solve the problem. Identification of the possible forms of the module presentation.

3. Project activity: active students' self-organization activities. Teachers counseling. Formalizing the obtained results.

\subsection{The presentation of the results.}

A presentation is a visual representation of what was the objective and what has been achieved in the joint resolution of the problem. While making a presentation one must clearly and vividly show the result of the students' creative activity as well as summarize the process, the difficulties arisen, the ways they were solved. At this stage, the students summarize the urgency of the issue, tasks and objectives, draw conclusions and reasonably argue for their opinion. They represent a module project, take into account the alternative views of others.

Types of presentations:

1. Oral report (it is possible to support the oral report with visual aids).

2. Theatrical performance.

3. Video.

4. Electronic media presentations (website, computers, etc. ).

5. Conferences.

\subsection{The registration of the results of the module implementation}

At the final stage the students can present either an abstract or presentation in Microsoft Power Point. For the abstract it is advisable to include no more than 10 pages of A4, 14 Times New Roman font line spacing 1. 0 , and for the presentation of no more than 12 slides.

The teacher shows a sample of the title page. The cover sheet is followed by the table of contents. The introduction should contain a justification of theoretical and pragmatic significance of the work, its relevance, provide an overview of the literature, define the problem, formulate the objectives and tasks necessary to implement the module. The central section reveals the contents of the activities, characteristics of the phenomenon or process, analyzes the key concepts. Relying on the studied materials the students should independently carry out a comprehensive research, systematize and interpret information. In the conclusion, the students give brief statements of the results obtained in the form of compressed, logically completed characteristics, analyze the data, underline the disputable questions and outline the research directions for the future.

\subsection{The materials for self-study}

The recommended literature is compulsory, optional, internet resources, periodicals. 


\section{Discussions}

The productivity of implementation of the interdisciplinary teaching modules in liberal education of the students as a practical mechanism of implementing the synergetic approach into educational practice of higher school is confirmed by the results of experimental use of the modules in the course of Fundamentals of Philosophy (the philosophical culture of the specialist's personality), Pedagogy (the methodological culture of the teacher), in National history (Cultural Identity of the personality of a modern specialist), in Literature (cultural and liberal universals in the works of the XXth century writers). The criteria for the dynamics extent of the synergetic values of personal self-organization in the process of studying the interdisciplinary modules are supported by cognitive performance (logically expressed knowledge - 75\% of respondents), the formation of critical thinking (emotional component) - 53\%; eagerness to continuous self-improvement and self-transformation (behavioral aspect) - 76\%; formation of professional competence (social and professional component) - 78\%. The initial level of the studied factors ranged from $35-39 \%$.

\section{Conclusion}

The research results of the synergetic approach to liberal education in higher school shows that the humanities as well as science-based, technical education receive new milestones to identify the underlying, essential foundations and connections between the various processes of the world, their combining into a single coherent structure. A strategic decision in this direction is including the cycles of academic courses in all vocational curricula, determining the constituent components of their basic concepts and categories in terms of interdisciplinary communication. The productivity and quality of the unified methodology of liberal education (language, terms, symbols) in modern conditions is determined exclusively by the ideas of synergetic approach, where the interdisciplinary modules act as the practical mechanism of implementation.

\section{References}

Andreev, V. I. (1996). Pedagogy of creative self-development. Kazan.

Boguslavskiy, M. V. (1999) The Passion for synergy. Mir obrazovanija, 5, 17 - 21.

Budanov, V. G. (2013). The synergy methodology in postnonclassical science and education. Moscow, 164-197.

Fedorova, M. A. (2002). The principles of self-organization theory in the education process. VI International scientific-methodical conference. Penza, 317 - 319

Haken, G. (1980). Synergy. Moscow.

Knyazeva, E. N. (1991). An accident, that creates the world (new concepts of self-organization in nature and society). Moscow, 34 - 40.

Makhmutov, M. I., Ibragimov G. I, Choshanov, M. A. (1993) The educational technologies of developing the students' thinking. Kazan, 10 $-28$.

Novikov, A. M. (2000). Russian education in the new era. The paradoxes of heritage, Moscow.

Prigozhin, I. , Stengers, I. (1986). The Order out of Chaos: Man's New Dialogue with Nature. Moscow.

Talanchuk, N. M, (1996). System-synergetic philosophy and concept of neo-Pedagogy: the stratagems of the pedagogical theory and practice development. Kazan.

Vinenko, V. G. (2001). System-synergetic modeling in teacher's continuous education. (Doctoral dissertation). Saratov.

Vyugina, S. V. (2015). Modernization of the educational system of the technological university in the development of the students' intellectual potential. Kazan, 185 - 189.

Zapesotskiy, A. S. (2003). Education: philosophy, culturology, policy. Moscow.

Zeer, E. F. (2000). Psychology of personality-oriented professional education. Ekaterinburg. 